CLINICAL CASE

\title{
INCREASED NUCHAL TRANSLUCENCY IN A FETUS WITH A NORMAL KARYOTYPE: CASE REPORT
}

\author{
Cristina Moisei ${ }^{1}$, Anca Lesnic ${ }^{1}$, Romina-Marina Sima ${ }^{1,2}$, Liana Pleş ${ }^{1,2}$ \\ ${ }^{1}$ Clinical Hospital "Sf. Ioan", "Bucur" Maternity, Bucharest, Romania \\ ${ }^{2}$ Carol Davila University of Medicine and Pharmacy, Bucharest, Romania
}

Corresponding author: Romina-Marina Sima

Email: romina.sima@yahoo.es

\begin{abstract}
Nuchal translucency (NT) is the normal fluid filled subcutaneous space measured at the back of the fetal neck measured in the late first trimester and early second trimester. Nuchal translucency screening can detect approximately $80 \%$ of fetuses with Down syndrome and other major aneuploidies with a rate of 5\% of false positive results, but the merger of the NT screening with $\beta-h C G$ and PAPP-A testing increases the detection rate to $90 \%$. We present the case of a fetus with a NT of $49 \mathrm{~mm}$ detected at the first trimester ultrasound morphologic exam. The Kryptor test revealed a 1:35 risk for Trisomy 13 and 1:721 for Trisomy 18. We report the case of an investigated pregnancy with a NT of $49 \mathrm{~mm}$ detected at the first trimester ultrasound exam, with a risk of 1:35 for Trisomy 13 and 1:721 for Trisomy 18 calculated at the Kryptor test. A chorionic villus sampling was recommended and performed with a result of $46 X Y$ normal karyotype. The particularity of this case is represented by the increased nuchal translucency as well as an increased risk for trisomy 13 and 18 in a normal karyotype fetus that had a normal development in the second and third trimester with no pregnancy complications arising.
\end{abstract}

KEYWORDS: Kryptor test, trisomy 13, trisomy 18, chorionic villus sampling, karyotype test

\section{INTRODUCTION}

Nuchal translucency (NT) is the normal fluid filled subcutaneous space measured at the back of the fetal neck measured in the late first trimester and early second trimester. In fetuses with genetic defects, cardiac anomalies and chromosomal abnormalities we can observe an increased nuchal translucency. Nuchal translucency screening can detect approximately $80 \%$ of fetuses with Down syndrome and other major aneuploidies with a rate of $5 \%$ of false positive results, but the merger of the NT screening with $\beta$-hCG and PAPP-A testing increases the detection rate to $90 \%$ [1]. There are new studies that show that the rate of detection can be increased to $95 \%$ examining the length of the nasal bone, the tricuspid and ductus venosus blood flow [2].Studies have shown that a NT of $3 \mathrm{~mm}$ in late first trimester carries a 4-fold risk for fetal trisomies, and a NT greater than $3 \mathrm{~mm}$ a 29-fold risk, even if the fetus has a normal karyotype, furthermore a NT of $4 \mathrm{~mm}$ or more is associated strongly with poor pregnancy outcomes [3]. 

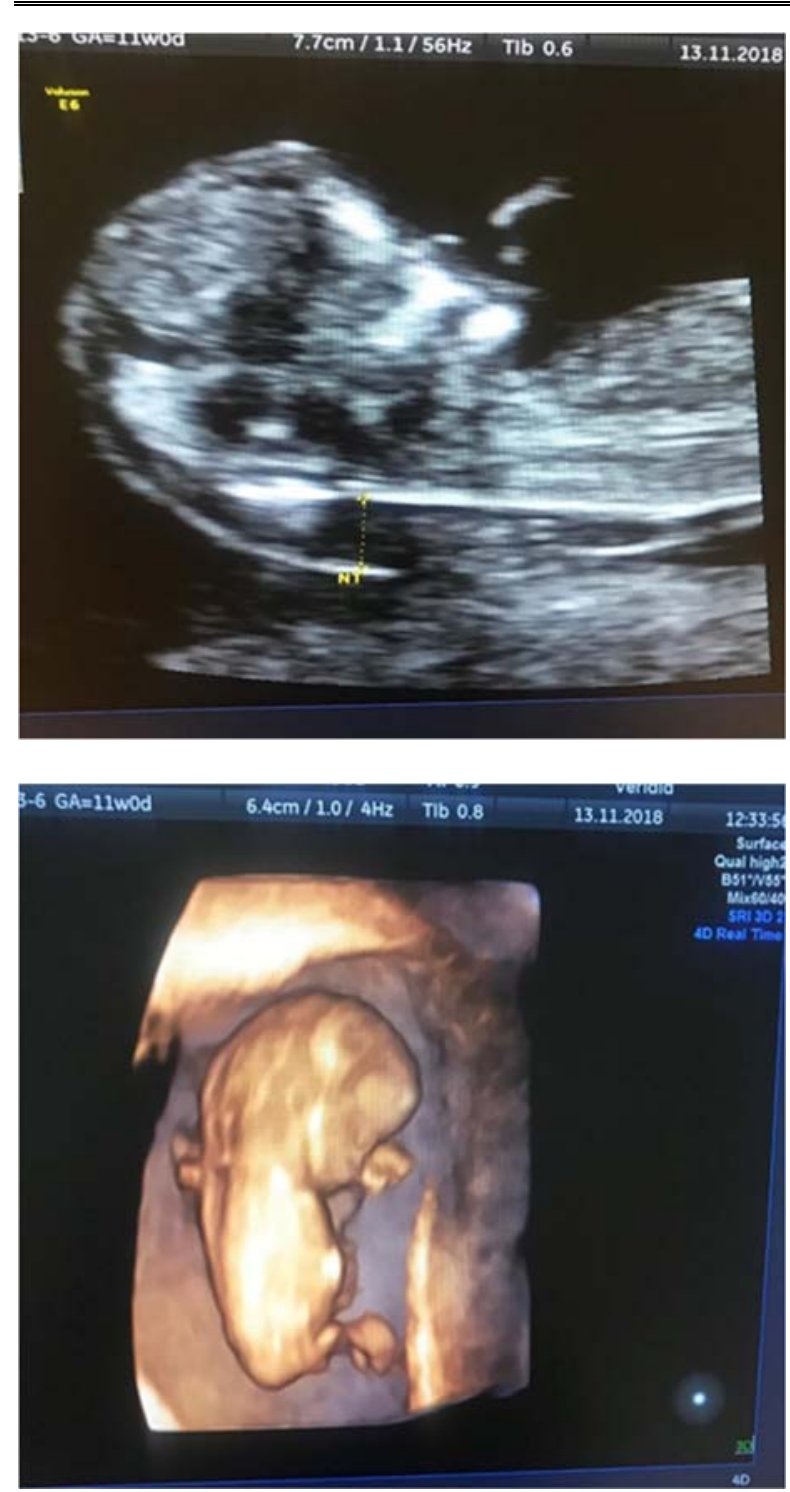

Figure 1 and 2 - Ultrasound image of an embryo at 11 weeks of gestation, the measured NT is 49 $\mathrm{mm}$. The second image is of a reconstructed 3D image of the same embryo.

The ultrasonographic evaluation of the NT was first described in 1985 by Benacerraf et al [3].

Nuchal fold thickness (NFT, measured between 16 and 24 weeks of gestation) has been accepted as markers for Down syndrome, with a normal value of less than $6 \mathrm{~mm}$ in multiple studies [4]-[6].

The protocol for the NFT assessment is strictly regulated, because the overestimation of the NFT can lead to an increase in invasive diagnostic tools like amniocentesis and chorionic villus sampling. The measurement should be made at the transcerebellar section level, having cavum septum pellucidum, the cerebral peduncles, the cerebellar peduncles and cisterna magna as ultrasonographic landmarks $[4],[7]$.

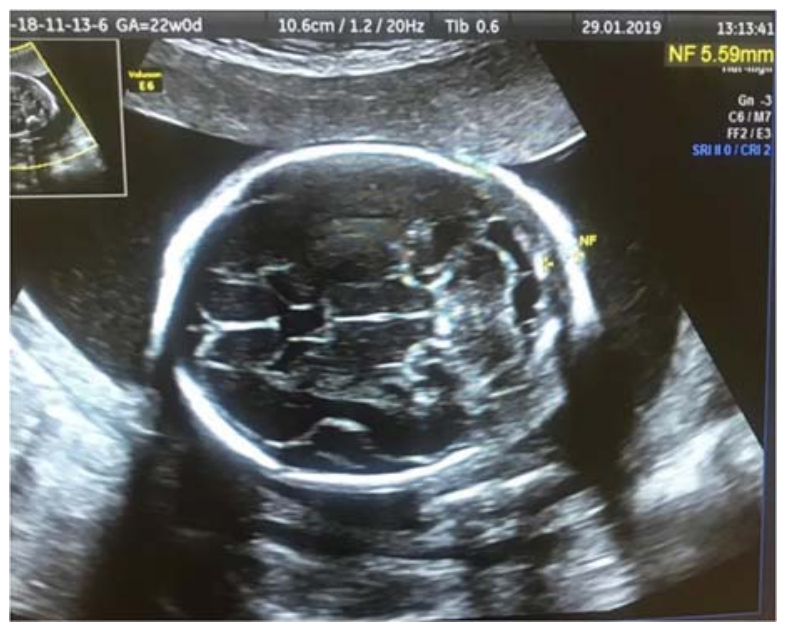

Figure 3 - Transcerebellar section, NFT of 5,59 mm (the measured NT in the first trimester was $4.9 \mathrm{~mm}$ - Figure 1).

\section{MATERIALS AND METHODS}

We present the case of a fetus with a NT of $49 \mathrm{~mm}$ detected at the first trimester ultrasound morphologic exam. The Kryptor test revealed a 1:35 risk for Trisomy 13 and 1:721 for Trisomy 18.

\section{RESULTS}

We report the case of an investigated pregnancy with a NT of $49 \mathrm{~mm}$ detected at the first trimester ultrasound exam, with a risk of 1:35 for Trisomy 13 and 1:721 for Trisomy 18 calculated at the Kryptor test. A chorionic villus sampling was recommended and performed with a result of 46XY normal karyotype. The second trimester ultrasound morphologic exam showed a normal NFT of $5.59 \mathrm{~mm}$, with a normal development, no major cardiac anomalies visible. The pregnancy was supervised by an OBGYN specialist, no maternal pathology present.

\section{DISCUSSIONS}

Although the implementation of the nuchal translucency (NT) evaluation in 1992 by Nicolaides et al was a major medical advance in the screening for chromosomal aneuploidies in the first trimester, other pathological conditions 
besides chromosomal aneuploidies are also linked with increased NT, but there are also the cases of normal fetuses with increased NT [8].

A study on a group of patients from Iasi, Romania concluded that even though it's a common practice to indicate an amniocentesis or a chorionic villus sampling in fetuses with an increased NT the majority of cases with increased NT in the study did not involve the presence of an aneuploidy, furthermore the pregnancy result in a positive outcome if no other anomaly is present, but the need for larger multicenter studies is pressing [8].

Another study published in 2015 concluded that there was no link between first trimester nuchal translucency measurements and complications that could develop in pregnancy [9].

Multiple studies concluded that negative pregnancy outcome increased with increasing NT, even if it is a minimal increase, even in fetuses with normal karyotype, but the overall prognosis is good if the second trimester ultrasound screening is negative for fetal abnormalities, the most frequent one detected in fetuses with increased NT being cardiac defects [10], [11].

\section{CONCLUSIONS}

The particularity of this case is represented by the increased nuchal translucency as well as an increased risk for trisomy 13 and 18 in a normal karyotype fetus that had a normal development in the second and third trimester with no pregnancy complications arising.

\section{REFERENCES}

[1]Padula, Francesco et al. "Retrospective study evaluating the performance of a first-trimester combined screening for trisomy 21 in an Italian unselected population." Journal of prenatal medicine vol. 8,3-4 (2014): 50-6.

[2]Geipel A, Willruth A, Vieten J, Gembruch U, Berg C. Nuchal fold thickness, nasal bone absence or hypoplasia, ductus venosus reversed flow and tricuspid valve regurgitation in screening for trisomies 21, 18 and 13 in the early second trimester. Ultrasound Obstet Gynecol 2010;35:535-9.

[3]Pandya, PP, Brizot, ML, Kuhn, P, Snijders, RJ, Nicolaides, KH. First-trimester fetal nuchal translucency thickness and risk for trisomies Obstet Gynecol. 1994 Sep;84(3):420-3.

[4]Benacerraf, BR, Barss, VA, Laboda, LA. A sonographic sign for the detection in the second trimester of the fetus with Down's syndrome. Am J Obstet Gynecol 1985; 151: 1078- 1079.

[5]Benacerraf, BR, Frigoletto, FD, Laboda, LA. Sonographic diagnosis of Down syndrome in the second trimester. Am J Obstet Gynecol 1985; 153: $49-52$.

[6]Benacerraf, BR, Frigoletto, FD, Cramer, DW. Down syndrome: sonographic sign for diagnosis in the second-trimester fetus. Radiology 1987; 163: 811-813.

[7]Crane, JP, Gray, DL. Optimal nuchal skin-fold thresholds based on gestational age for prenatal detection of Down syndrome. Am J Obstet Gynecol 1994; 171: 1282- 1286.

[8]Socolov, D., Socolov, R., Gorduza, V. E., Butureanu, T., Stanculescu, R., Carauleanu, A., \& Pavaleanu, I. (). Increased nuchal translucency in fetuses with a normal karyotype-diagnosis and management: An observational study. Medicine, 96(29), e7521. doi:10.1097/MD.0000000000007521

[9]Uysal, F., Cosar, E., Yucesoy, K., et al. Is there any relationship between adverse pregnancy outcome and first trimester nuchal translucency measurements in normal karyotype fetuses? J Matern Fetal Neonatal Med 2014;27:1-4.

[10]Lithner, C. U., Kublickas, M., \& Ek, S. Pregnancy outcome for fetuses with increased nuchal translucency but normal karyotype. Journal of Medical Screening, 2016. 23(1), 1-6. https://doi.org/10.1177/0969141315595826

[11]Äyräs, O. , Tikkanen, M. , Eronen, M. , Paavonen, J. and Stefanovic, V. Increased nuchal translucency and pregnancy outcome: a retrospective study of 1063 consecutive singleton pregnancies in a single referral institution. Prenat Diagn, (2013), 33: 856-862. doi:10.1002/pd.4143. 